\title{
Large Amplitude Dust-acoustic Solitons in a Plasma Consisting of Super- thermal Electron-positron Pairs and Dust Particulates
}

\author{
E. Saberian ${ }^{1, *}$, A. Esfandyari-Kalejahi ${ }^{2}$ and M. Afsari-Ghazi ${ }^{2}$ \\ ${ }^{I}$ Department of Physics, Faculty of Basic Sciences, University of Neyshabur, P.O. Box 91136-599, Neyshabur, Iran \\ ${ }^{2}$ Department of Physics, Faculty of Sciences, Azarbaijan Shahid Madani University, P.O. Box 53714-161, Tabriz, Iran
}

\begin{abstract}
The occurrence and propagation of large amplitude dust-acoustic solitary waves (DASWs) are studied in a three-component plasma consisting of negatively charged dust grains and electron-positron pairs by employing a pseudopotential technique. Here, we focus on a superthermal plasma modeled by a $\kappa$-like distribution and consider a finite temperature for dust particulates. It is shown that the solitary waves with negative polarity are allowed in the system and there is a critical value for dust charge $Z_{d}$ above/below which the subsonic/supersonic solitary structures can propagate. In the case of negative fullerene ions, $Z_{d}=1$, it is observed that subsonic DASWs can propagate in the plasma. In addition, it is revealed that the propagation of double layers is not possible in this plasma system.
\end{abstract}

Keywords: Ion-acoustic solitary wave, Kappa distribution, Superthermal particle, Sagdeev pseudo-potential.

\section{INTRODUCTION}

In recent years, dusty plasmas have attracted a great deal of attention due to their ubiquitous nature. Dusty plasmas may occur in several situations related to the astrophysical environment such as cometary tails, asteroid zones, planetary rings, interstellar medium, nebulas, earth's ionosphere and magnetosphere [1-5]. Electron-positron-dust (e-p-d) plasma is a kind of dusty plasma in which electrons and positrons have equal masses. Such plasmas can occur in supernovae and pulsar environments, as well as in cluster explosions by intense laser beams in laboratory experiments. The production of electron-positron (EP) pairs in supernovae and pulsar magnetosphere is significant, where the environment include large quantities of EP pairs, as well as dust particles [6]. Clusters, on the other hand, are produced as bonded atomic structures in laboratory to form nanoscale plasmas by intense laser fields. These plasmas have a life span of the order of femtoseconds, after which they erupt in a release of highly energetic electrons and dust particles [7-9]. On the other hand, the production of EP pairs and dust particles due to cluster explosions can be possible by the next generation lasers [10].

Nowadays, it is believed that the study of nonlinear waves in space plasmas improves our knowledge about the earth's magnetosphere and the other plasma environment. Generally, small-amplitude nonlinear waves in plasma can be described either by the Korteweg-de Veries (KdV) equation or nonlinear Schrödinger equation (NLSE), derived via

*Address correspondence to this author at the Department of Physics, Faculty of Basic Sciences, University of Neyshabur, P.O. Box 91136-599, Neyshabur, Iran; Tel: (+98)5142628000; Fax: (+98)5412629001;

E-mail: esaberyan@yahoo.com reductive perturbation method [11], but the nonlinear waves with arbitrary amplitude can be analyzed by using the standard Sagdeev pseudo-potential approach [12]. For example, dust-acoustic solitons of large amplitude with variable particle charge are studied using the Sagdeev quasi-potential analysis by Ivlev and Morfill [13]. Moreover, the characteristics of solitons in Earth's dusty mesosphere have been examined by Popel et al. [14]. Furthermore, because of the coexistence of EP pairs and dust grains in many astrophysical environment, there are some theoretical studies about nonlinear wave propagation in e-p-d plasmas, see for example [15-17]. It is found that the presence of charged dust grains modifies the existing plasma wave spectra and may even introduce new eigenmodes in the plasma. In the lowfrequency limit the dust motion has to be taken into account, which leads to dust-acoustic (DA) modes [18-20].

Most of studies on the waves in plasmas are based on the assumption of a Maxwellian distribution function being the most probable distribution function for multicomponent species [21-25]. However, with more and more empirical data becoming available from space plasma systems, it is realized that in such systems, the particle distribution significantly deviates from the Maxwellian distribution [26-28] due to the presence of superthermal particles having high energy tails [29]. These superthermal particles can be described by $\kappa$ distribution rather than Maxwellian [30-38]. The Lorentzian (or Kappa) distribution has been widely used for conventional plasmas (not for a dust component). The $\kappa$ distribution was first suggested by Vasyliunas [29] to model space plasmas, and was later adopted by many authors in various physical contexts. Superthermal (accelerated) particles are often present in laboratory experiments in the solar atmosphere [39] and in space plasma [30] environment. The parameter 
$\kappa$ determines the high energy power law index (which approaches to Maxwellian distribution for $\kappa \rightarrow \infty$ ) [40]. The $\kappa$ distribution function was argued to provide a much better fit to existing observations than the Maxwellian [29-41]. The kappa distribution function applications include; for instance, the interpolation of observations in the Earth's foreshock with $3<\kappa_{e}<6[42,43]$ and the solar wind models with coronal electrons with $2<\kappa_{e}<6[42,44]$.

The commonly used three-dimensional, isotropic kappa $(\kappa)$ distribution is given by $[45,46]$;

$$
f_{\kappa}(v)=\frac{n_{0}}{\left(\pi \kappa v_{\text {eff }}^{2}\right)^{\frac{3}{2}}} \frac{\Gamma(\kappa+1)}{\Gamma\left(\kappa-\frac{1}{2}\right)}\left(1+\frac{v^{2}}{\kappa v_{\text {eff }}^{2}}\right)^{-(\kappa+1)},
$$

where, $n_{0}$, is the equilibrium number density, $v_{\text {eff }}^{2}=(\kappa-3 / 2) / \kappa \times\left(2 K_{B} T / m\right)$ is the effective thermal speed modified by the spectral index $\kappa(>3 / 2)$ with $T$ being the temperature, $m$ the mass of species, and $\Gamma(x)$ is the gamma function. By using the kappa distribution function and integrating over velocity space, one may obtain the number density of the plasma species. It is emphasized that this "Kappa" distribution tends to a Maxwellian one for $\kappa \rightarrow \infty$.

As mentioned above, the $\kappa$ distribution function gives rise to much better fit to the experimental observations than the Maxwellian one [29, 41]. On the other hand, the e-p-d plasmas can occur in space plasmas as well as in laboratory plasmas. According to our knowledge, the study of dustacoustic solitary waves (DASWs) in the e-p-d plasmas consisting of superthermal electrons, positrons and dust particulates has not been done yet. Therefore, the aim of the present paper is the studying such structures in the e-p-d plasmas. One must notice that the EP annihilation time scale is very small compared with the time scale of DA waves. So, we assume that the recreation of EP pairs can be taken place by, for example, sunlight or intense laser radiation in DA wave time scale.

The paper is organized as follows: in Section 2, the basic set of fluid equations is presented and reduced in Section 3 to an energy balance equation, which defines a pseudopotential model of the nonlinear DA excitations. In Section 4, we define conditions for the existence of large amplitude DASWs and illustrate the dependence of existence region of these modes on some plasma parameters. Finally, Section 5 is devoted to conclusions.

\section{THE MODEL EQUATIONS}

Let us consider a collisionless plasma comprising of a population of superthermal distributed electrons and positrons in the background of extremely massive inertial dust grains which are negatively charged. Therefore, in one-dimension, the nonlinear dynamics of DAWs in e-p-d plasma is governed by the following system of fluid equations;

$$
\begin{aligned}
& \frac{\partial n_{d}}{\partial t}+\frac{\partial}{\partial x}\left(n_{d} v_{d}\right)=0, \\
& \frac{\partial v_{d}}{\partial t}+v_{d} \frac{\partial v_{d}}{\partial x}=\frac{e Z_{d}}{m_{d}} \frac{\partial \Phi}{\partial x}-\frac{1}{n_{d} m_{d}} \frac{\partial p_{d}}{\partial x}
\end{aligned}
$$

and the Poisson's equation;

$$
\frac{\partial^{2} \Phi}{\partial x^{2}}=4 \pi e\left(n_{e}+Z_{d} n_{d}-n_{p}\right)
$$

where, $\mathrm{n}_{\mathrm{d}}, n_{e}$ and $n_{p}$ refer to the number density of dust particles, electrons and positrons, respectively; $v_{d}$ is the velocity of dust fluid; $p_{d}$ is the pressure of dust fluid; $Z_{d}$ is the atomic number of dust particulates and $\Phi$ is the electrostatic potential. The pressure $p$ is related to the density $n$ by the thermodynamic equation of state $p / n^{\gamma}=$ const. , where $\gamma$ is the polytropic index or the ratio of specific heats $C_{p} / C_{v}$. By using the thermodynamic equation of state, we may find an explicit expression for compression of the species we are dealing with as $\frac{\vec{\nabla} p}{p}=\gamma \frac{\vec{\nabla} n}{n}$. In the present work in which we are studying the low-frequency DA waves, the electrons (or positrons) move so fast relative to the wave that they have sufficient time to equalize their temperature everywhere; it means that the electrons (or positrons) are isothermal. For these isothermal particles we have $\vec{\nabla} p=\vec{\nabla}\left(n k_{B} T\right)=k_{B} T \vec{\nabla} n$ which leads the value $\gamma_{e(p)}=1$ for them. On the other hand, it is assumed that the dust particles suffer one-dimension compressions in the plane wave. These heavy particles have not the time to equalize their temperature everywhere in the wave motion and so $k_{B} T_{d}$ will change in the compression process. So we have a pressure-gradient as $\vec{\nabla} p_{d}=\vec{\nabla}\left(n_{d} k_{B} T_{d}\right)=k_{B} T_{d} \vec{\nabla} n_{d}+n_{d} \vec{\nabla} k_{B} T_{d}$, combining with the equation of state result $\gamma_{d}$ would have a value larger than one; it means that the dust particles have adiabatic compressions. Generally, if $N$ is the number of degrees of freedom, $\gamma$ is given by $\gamma=(2+N) / N$ [47]. It is to be mentioned that we have essentially assumed that the heat flow in the system is negligible in order to have an equation of state like $p / n^{\gamma}=$ const. In the present study, we have to take $\gamma_{d}=3$ for one-dimension adiabatic compressions for dust particles $(N=1)$.

We emphasize that the dust particle charge depends generally on the electrostatic potential and hence, in different 
places of the soliton the dust charge should be different. The variation of dust charge is because of collisions between electrons and positrons with dust particles. In practice, the collision of electrons and positrons with dust grains produces a charged current. The time evolution of charge on a dust grain immersed in a plasma is determined by the charging equation as follows;

$$
\left(\frac{\partial}{\partial t}+\vec{v}_{d} \cdot \vec{\nabla}\right) Q_{d}=\mathrm{I}_{e}+\mathrm{I}_{p}
$$

where $\mathrm{I}_{e}$ and $\mathrm{I}_{p}$ are, respectively, the currents which charges the dust grain via electrons and positrons, reaching the grain surface. The currents $\mathrm{I}_{e}$ and $\mathrm{I}_{p}$ are given by following relations [48];

$$
\begin{aligned}
& \mathrm{I}_{e}=-e \pi r_{d}^{2}\left(\frac{8 T_{e}}{\pi m_{e}}\right)^{2} n_{e} \exp \left(\frac{e \phi}{T_{e}}\right) \\
& \mathrm{I}_{p}=+e \pi r_{d}^{2}\left(\frac{8 T_{P}}{\pi m_{P}}\right)^{2} n_{p} \exp \left(\frac{-e \phi}{T_{e}}\right)
\end{aligned}
$$

where $r_{d}$ is the radius of dust grains, and $\phi$ denotes the dust grain surface potential relative to the plasma potential $\Phi$. In many cases, the dust charge can quickly reach the local equilibrium at which the currents from the electrons and positrons to the dust grains are balanced. We further suppose that the streaming velocities of electrons and positrons are much smaller than the thermal velocities. So, the charge-current balance equation reduce to $\mathrm{I}_{e}+\mathrm{I}_{p} \approx 0$.

In the present work, we are interested in examining the nonlinear propagation of the low phase velocity electrostatic modes (in comparison with the electron and positron thermal speeds) on the time scale of the DA wave period. So, by considering the situations in which the typical dust charging time scale is longer than DA time scale, it is anticipated that the dust charge fluctuations have no essential effect on DA modes [49]. So, we can assume that the dust charge inside the solitons is constant.

Following Gill et al. [50], the number densities of electrons and positrons are expressed as:

$$
\begin{aligned}
& n_{e}=n_{e 0}\left(1-\frac{e \Phi}{\left(\kappa-\frac{3}{2}\right) K_{B} T_{e}}\right)^{-\kappa+\frac{3}{2}} \\
& n_{p}=n_{p 0}\left(1+\frac{e \Phi}{\left(\kappa-\frac{3}{2}\right) K_{B} T_{p}}\right)^{-\kappa+\frac{3}{2}}
\end{aligned}
$$

The right hand side of Eq. (3) cancels at equilibrium due to the overall neutrality condition, then:

$n_{p 0}=n_{e 0}+Z_{d} n_{d 0}$.
We define the DA speed as $c_{d}=\left(K_{B} T_{e} / m_{d}\right)^{1 / 2}$ and take the Debye length $\lambda_{D}$ given by the formula $1 / \lambda_{D}^{2}=\sum_{j} 4 \pi n_{j 0} q_{j}^{2} / K_{B} T_{j}(j=e, p)$, where $q_{j}$ denotes the charge of electron or positron. Now, we may normalize the time $t$ by the inverse of dust plasma-frequency $\omega_{p d}^{-1}=\left(m_{d} / 4 \pi Z_{d}^{2} e^{2} n_{d 0}\right)^{1 / 2}$, the distance $x$ by $\lambda_{D}$, the pressure $p_{d}$ by $p_{d 0}=n_{d 0} K_{B} T_{d}$, the potential $\Phi$ by $\Phi_{0}=K_{B} T_{e} / e Z_{d}$, the velocity $v_{d}$ by DA speed $c_{d}$, and finally $n_{e, p, d}$ by $n_{d 0}$. So, the nonlinear dynamics of the lowfrequency DA waves in our dusty plasma is governed by the following reduced equations;

$$
\begin{aligned}
& \frac{\partial n_{d}}{\partial t}+\frac{\partial}{\partial x}\left(n_{d} v_{d}\right)=0 \\
& \frac{\partial v_{d}}{\partial t}+v_{d} \frac{\partial v_{d}}{\partial x}=\frac{\partial \Phi}{\partial x}-3 \sigma_{d e} n_{d} \frac{\partial n_{d}}{\partial x}
\end{aligned}
$$

$$
\frac{\partial^{2} \Phi}{\partial x^{2}}=\beta_{1}\left\{\begin{array}{l}
\frac{\beta_{e d}}{Z_{d}}\left(1-\frac{\Phi}{\left(\kappa-\frac{3}{2}\right) Z_{d}}\right)^{-\kappa+\frac{1}{2}} \\
+n_{d}-\frac{\beta_{p d}}{Z_{d}}\left(1+\frac{\Phi}{\left(\kappa-\frac{3}{2}\right) Z_{d} \sigma_{p e}}\right)^{-\kappa+\frac{1}{2}}
\end{array}\right\},
$$

where we have defined the fractional temperatures as $\sigma_{d e}=T_{d} / T_{e}$ and $\sigma_{p e}=T_{p} / T_{e}$, in which $T_{e}, T_{p}$ and $T_{d}$ refer to the temperature of electrons, positrons and dust particles, respectively. Furthermore $\beta_{e d}=n_{e 0} / n_{d 0}$ and $\beta_{p d}=n_{p 0} / n_{d 0}$ refer to the ratios of unperturbed densities of electron to dust and positron to dust, respectively, and also the parameter $\beta_{1}$ is given by $\beta_{1}=\frac{Z_{d}^{2}}{\beta_{e d}+\beta_{p d} / \sigma_{p e}}$. It is to be noted that the right-hand side of Eq. (11) cancels at equilibrium due to the overall neutrality condition and yield the following result:

$$
\beta_{p d}=\beta_{e d}+Z_{d}
$$

\section{THE EXACT NONLINEAR FORMALISM}

To obtain a solitary wave solution, we assume that all fluid variables in the evolution equations depend on a single variable $\xi=x-M t$, where $M$ is the Mach number (the velocity of the solitary wave normalized by DA speed 
$c_{d}$ ). Appling appropriate boundary conditions for localized waves: i.e., $\Phi \rightarrow 0, v_{d} \rightarrow 0, n_{d} \rightarrow 1$ as $\xi \rightarrow \pm \infty$, and then integrating Eqs. (9) and (10), we obtain:

$n_{d}=\frac{M}{M-v_{d}}$,

$3 \sigma_{d e} n_{d}^{4}-n_{d}^{2}\left(M^{2}+2 \Phi+3 \sigma_{d e}\right)+M^{2}=0$.

From Eq. (14), two solutions are obtained for the dust density $n_{d}$ as follows:

$n_{d \pm}=\frac{1}{\sqrt{6 \sigma_{d e}}}\left\{M^{2}+3 \sigma_{d e}+2 \Phi \pm \sqrt{\begin{array}{l}\left(M^{2}+3 \sigma_{d e}+2 \Phi\right)^{2} \\ -12 \sigma_{d e} M^{2}\end{array}}\right\}^{\frac{1}{2}}$.

The difference between two solutions implies an inspection in terms of the interplay between the values of parameters $M, \sigma_{d e}$ and $\Phi$. Three criteria need to be considered here;

(i) First, the reality of $n_{d}$ needs to be ensured.

(ii) Furthermore, one has to impose $n_{d} \rightarrow 1$ as $\Phi \rightarrow 0$, i.e. the equilibrium state must be accessible as expected.

(iii) Finally, $n_{d}$ should be analytical (non-singular) for all positive values of $\sigma_{d e} \geq 0$. In fact, it should tend smoothly to the correct cold dust density $|M| /\left(M^{2}+2 \Phi\right)^{1 / 2}$ in the limit $\sigma_{d e} \rightarrow 0$. We find that $n_{d-}$ satisfies these criteria only if;

(a) $|M|>\sqrt{3 \sigma_{d e}}$ (necessary for $n_{d-} \rightarrow 1$ as $\Phi \rightarrow 0$ ) and

(b) $\Phi>\frac{-1}{2}\left(|M|-\sqrt{3 \sigma_{d e}}\right)^{2} \equiv \Phi_{1}$ (which is necessary because $\mathrm{n}_{\mathrm{d}-}$ must be real).

Note that the cold-dust limit is smoothly recovered upon setting $\sigma_{d e}=0$. On the other hand, we find that $\mathrm{n}_{\mathrm{d}_{+}} \rightarrow 1$ as $\Phi \rightarrow 0$ if the inequality $|M|<\sqrt{3 \sigma_{d e}}$ is satisfied. However, since $\mathrm{n}_{\mathrm{d}_{+}}$is not analytical for $\sigma_{d e} \rightarrow 0$, we shall therefore abandon this solution.

Introducing the ansatz (see Ref. [51]);

$\theta=\exp \left(\cosh ^{-1}(\chi)\right)$

where $\chi=\frac{M^{2}+3 \sigma_{d e}+2 \Phi}{|M| \sqrt{12 \sigma_{d e}}}$

the expression (15) for the dust density, $n_{d \pm}$, may now be written in the form: $n_{d \pm}=\frac{|M|^{1 / 2}}{\left(3 \sigma_{d e}\right)^{1 / 4}} \theta^{ \pm \frac{1}{2}}$

Note that the inverse-hyperbolic-cosine function is defined for values of the argument larger than zero $(\chi>0)$. Indeed, this is ensured by the reality of $n_{d \pm}$ given in Eq. (15).

By substituting $n_{d-}$ from Eq. (18) into the Poisson's Eq. (11), multiplying the resulting equation by $d \Phi / d \xi$, then integrating and applying the boundary condition $\Phi \rightarrow 0$ and $d \Phi / d \xi \rightarrow 0$ at $|\xi| \rightarrow \infty$, we find the following energybalance equation;

$\frac{1}{2}\left(\frac{d \Phi}{d \xi}\right)^{2}+\psi(\Phi, \mathrm{M})=0$,

where $\psi(\Phi, M)$ is the Sagdeev pseudo-potential. The Galilean transformation $\xi=x-M t$ is reminded, where $\xi$ is the distance in the commoving framework with the wave. The physical interpretation of the energy-balance Eq. (19) is that a fictitious particle (soliton) with position $\Phi$ and velocity $d \Phi / d \xi$ moves in the potential well $\psi(\Phi, M)$. The pseudopotential $\psi(\Phi, M)$ is given by;

$$
\begin{aligned}
& \psi(\Phi, M)=-\beta_{1}\left(3 \sigma_{d e}|M|^{6}\right)^{\frac{1}{4}}\left(\theta^{\frac{1}{2}}-\theta_{0}^{\frac{1}{2}}\right)- \\
& \frac{\beta_{1}}{3}\left(3 \sigma_{d e}|M|^{6}\right)^{\frac{1}{4}}\left(\theta^{-\frac{3}{2}}-\theta_{0}^{-\frac{3}{2}}\right) \\
& +\beta_{1} \beta_{e d}\left(1-\left(1-\frac{\Phi}{\left(\kappa-\frac{3}{2}\right) Z_{d}}\right)\right. \\
& +\beta_{1} \beta_{p d} \sigma_{p e}\left(1-\left(1+\frac{\Phi}{\left(\kappa-\frac{3}{2}\right) Z_{d} \sigma_{p e}}\right)^{-\kappa+\frac{3}{2}}\right),
\end{aligned}
$$

which is derived only for the $n_{d-}$ branch with $M^{2} \geq 3 \sigma_{d e}$. Here, the notation $\theta_{0}=\theta(\Phi=0)$ implies the unperturbed quantities.

\section{SMALL AMPLITUDE LIMIT}

Before proceeding to the large amplitude solitary waves, we consider the small-amplitude limit in the above analysis. 
By Taylor expanding the Sagdeev potential $\psi(\Phi, M)$ around $\Phi=0$ we obtain;

$\psi(\Phi, M) \approx \frac{\psi_{0}^{\prime \prime}}{2} \Phi^{2}+\frac{\psi_{0}^{\prime \prime \prime}}{6} \Phi^{3}$,

where we may calculate $\psi_{0}^{\prime \prime}=d^{2} \psi /\left.d \Phi^{2}\right|_{\Phi=0}$ and

$\psi_{0}^{\prime \prime \prime}=d^{3} \psi /\left.d \Phi^{3}\right|_{\Phi=0}$ from Eq. (20) as follows:

$\psi_{0}^{\prime \prime}=\frac{\beta_{1}}{M^{2}-3 \sigma_{d e}}-\frac{\kappa-\frac{1}{2}}{\kappa-\frac{3}{2}}$,

$\psi_{0}^{\prime \prime \prime}=-\frac{\beta_{1}}{Z_{d}^{3}} \frac{\kappa^{2}-\frac{1}{4}}{\left(\kappa-\frac{3}{2}\right)^{2}}\left(\beta_{e d}-\frac{\beta_{p d}}{\sigma_{p e}^{2}}\right)+$

$\frac{\beta_{1}}{3 \sigma_{d e}}\left(\frac{1}{M^{2}-3 \sigma_{d e}}-\frac{\left(M^{2}+3 \sigma_{d e}\right)^{2}}{\left(M^{3}-3 \sigma_{d e}\right)^{3}}\right)+\frac{\beta_{1}}{\left(M^{2}-3 \sigma_{d e}\right)^{2}}$.

Substituting Eq. (21) into Eq. (19) and integrating, we find a solitary solution in the form of:

$\Phi(\xi)=\frac{-3 \psi_{0}^{\prime \prime}}{\psi_{0}^{\prime \prime \prime}} \sec h^{2}\left(\frac{\sqrt{-\psi_{0}^{\prime \prime}}}{2} \xi\right)$.

The pulse profile given by (24) is identical to the solution of the Korteweg-de Vries (KdV) equation, which is obtained by the reductive perturbation method, (for example see Ref. [52], page 260). It is reminded that the soliton width $L=2 / \sqrt{-\psi_{0}^{\prime \prime}}$ and the amplitude $\Phi_{0}=-3 \psi^{\prime \prime} / \psi^{\prime \prime \prime}$ satisfy the relation $\Phi_{0} L^{2}=12 / \psi^{\prime \prime \prime}$, as we know from the standard $\mathrm{KdV}$ theory.

\section{CONDITIONS FOR OCCURRENCE OF DA SOLI- TONS: NUMERICAL ANALYSIS}

The existence of soliton-like excitations is characterized by inspection of the pseudo-potential $\psi(\Phi, M)$. It should be noted that $\psi(\Phi, M)$ is real when the inequality (16) is satisfied. Now, the conditions for existence of solitary wave structures are outlined below.

The first condition involves the curvature of the function $\psi(\Phi, M)$, which must have a maximum at $\Phi=0$. In addition to $\psi^{\prime}(\Phi=0)=\psi(\Phi=0)=0$ (here readily satisfied at equilibrium, as a result of the neutrality condition), we must have $\psi^{\prime \prime}(\Phi=0)<0$ (the primes denote differentiation). The latter condition implies the following inequality:

$\psi^{\prime \prime}(\Phi=0)=\frac{\beta_{1}}{M^{2}-3 \sigma_{d e}}-\frac{\kappa-\frac{1}{2}}{\kappa-\frac{3}{2}}<0$.
This relation shows the minimum possible values of the excitation speed (Mach number) $M$, at which localized pulses can propagate in the plasma.

Another conditions that we should apply them are as follows: $\psi(\Phi, M)<0$ in the interval $\left(\Phi_{m}, 0\right]$ and $\left[0, \Phi_{m}\right)$ and $d \psi /\left.d \Phi\right|_{\Phi=\Phi_{m}} \geq 0$, where $\Phi_{m}$ is the root of $\psi(\Phi)$, viz. $\psi\left(\Phi_{m}\right)=0$. For negative potential structures we have $\Phi_{m}=\Phi_{\min }<0$ and for positive potential structures we have $\Phi_{m}=\Phi_{\max }>0$. It is reminded that the (absolute value of the) abscissa where the curve intersects with the horizontal axis, $\Phi_{m}$, corresponds to the maximum amplitude of solitary waves. Here, it is understood that there are no other roots in between. These requirements are tantamount to imposing the requirement $\psi\left(\Phi_{c r}\right) \geq 0$, where the critical value $\Phi_{c r}$ is given by $\Phi_{c r}=\Phi_{1}=-1 / 2\left(|M|-\sqrt{3 \sigma_{d e}}\right)$ for negative potential structures (see Eq. (16)).

Now, in order to elucidate the characteristics of solitary waves structure involved in Eq. (19), we have numerically analyzed the pseudo-potential function $\psi(\Phi, M)$ and investigated the effects of some plasma parameters on existence regions of solitary waves and double layers, i.e.; the superthermal index $\kappa$, the charge of dust particles $Z_{d}$, and the fractional temperature of dust to electron, $\sigma_{d e}$, fractional temperature of positron to electron, $\sigma_{p e}$, and fractional density of electron to dust, $\beta_{\text {ed }}$.

Since the dust particles are much heavier than the electrons and positrons, the temperature of the dust particles have been considered much less than that of the electrons and positrons. Therefore, we have chosen a typical value as $\sigma_{d e}=0.01$. On the other hand, the electrons and positrons have the same mass and they can be in equilibrium with each other. Hence, we have chosen $\sigma_{p e}=1$. On the other hand, inspired with astrophysical observations, we have considered the spectral index $\kappa$ in the range $2<\kappa<6$ (see, e.g., Ref. [44]). It is to be mentioned that for values of the spectral index $\kappa$ above $\kappa \approx 10$, our results tends practically to that of a Maxwellian plasma.

(Figs. (1-3)) represent the allowed regions for the existence of dust-acoustic solitons in a subtle manner. Please note that these graphs are plotted in the plane $(\Phi, M) \Phi$, where

the solid curve indicates the contour of $\Phi=-(1 / 2)$ $\left(\mid M-\sqrt{3 \sigma_{d e}}\right)^{2}$. So, the regions above this contour indicate condition for reality of $n_{d-}$. We remind that only $n_{d-}$ satisfies the three criteria (i), (ii) and (iii), given in Sec. 3, and then result in the equation (16). On the other hand, in Figs. (1-3), we have plotted the contour $\psi(\Phi, M)=0$ with dotted, 


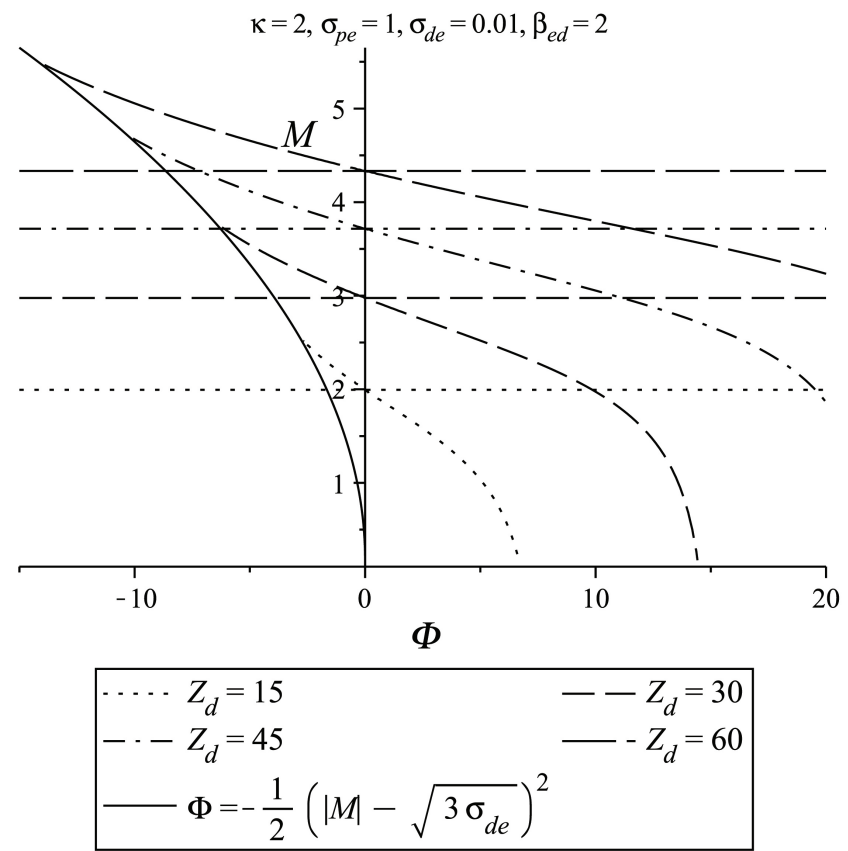

Fig. 1a

Fig. (1a). The contours of $\Phi=\frac{-1}{2}\left(|M|-\sqrt{3 \sigma_{d e}}\right)^{2}$ (solid curve), $\psi(\Phi)=0$ (doted, dashed, dot-dashed and long dashed curves for $Z_{d}=15, Z_{d}=30, Z_{d}=45$ and $Z_{d}=60$, respectively) and $\psi^{\prime \prime}(0)=0$ (doted, dashed, dot-dashed and long dashed horizontal lines for $Z_{d}=15, Z_{d}=30, Z_{d}=45$ and $Z_{d}=60$, respectively) in the $\Phi-M$ plane. The other plasma parameters set to $\kappa=2, \sigma_{p e}=1, \sigma_{d e}=0.01, \beta_{e d}=2$.

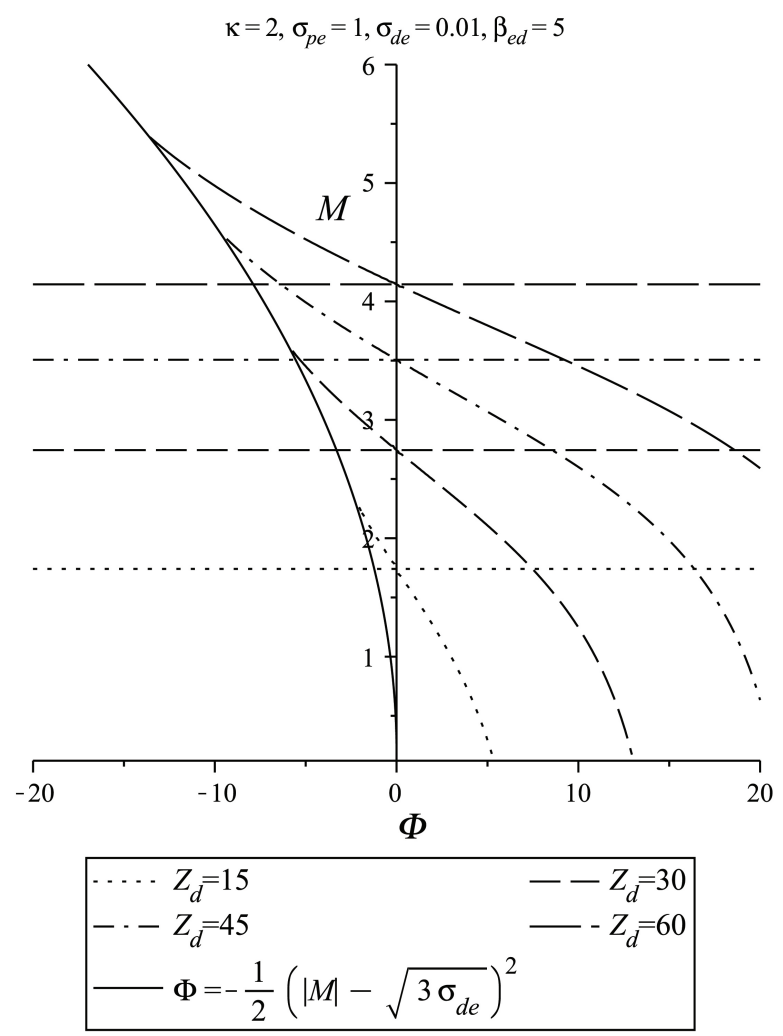

Fig. 1b

Fig. (1b). Same as in Fig. (1a), for $\kappa=2$ and $\beta_{e d}=5$. 


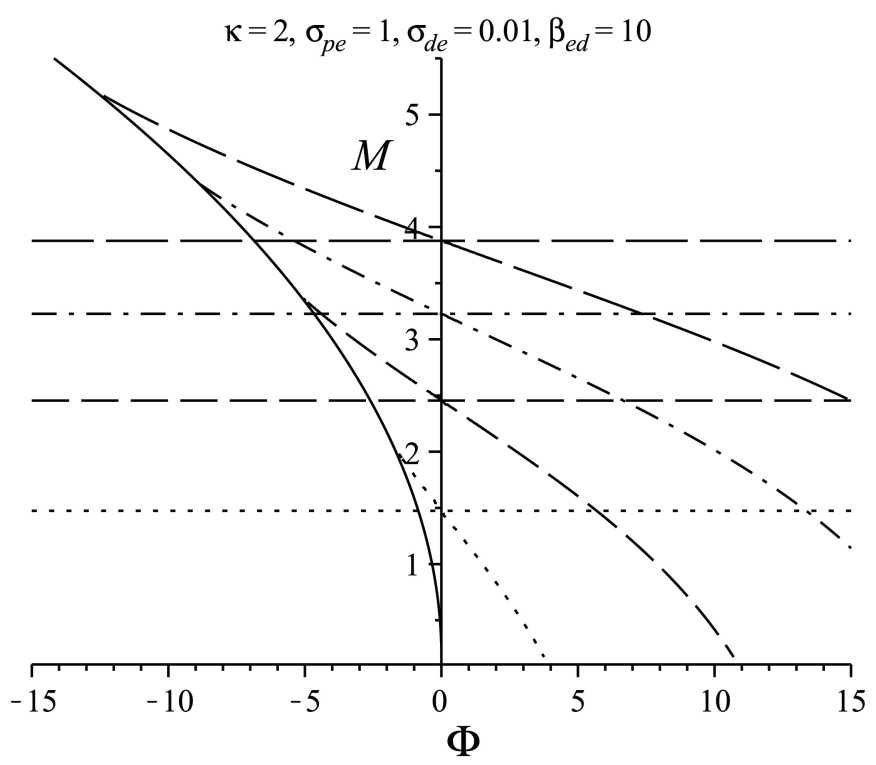

$$
\begin{array}{lr}
\cdots \cdots Z_{d}=15 & --Z_{d}=30 \\
--Z_{d}=45 & --Z_{d}=60 \\
-\Phi=-\frac{1}{2}\left(|M|-\sqrt{3 \sigma_{d e}}\right)^{2} &
\end{array}
$$

Fig. 1c

Fig. (1c). Same as in Fig. (1a), for $\kappa=2$ and $\beta_{e d}=10$.

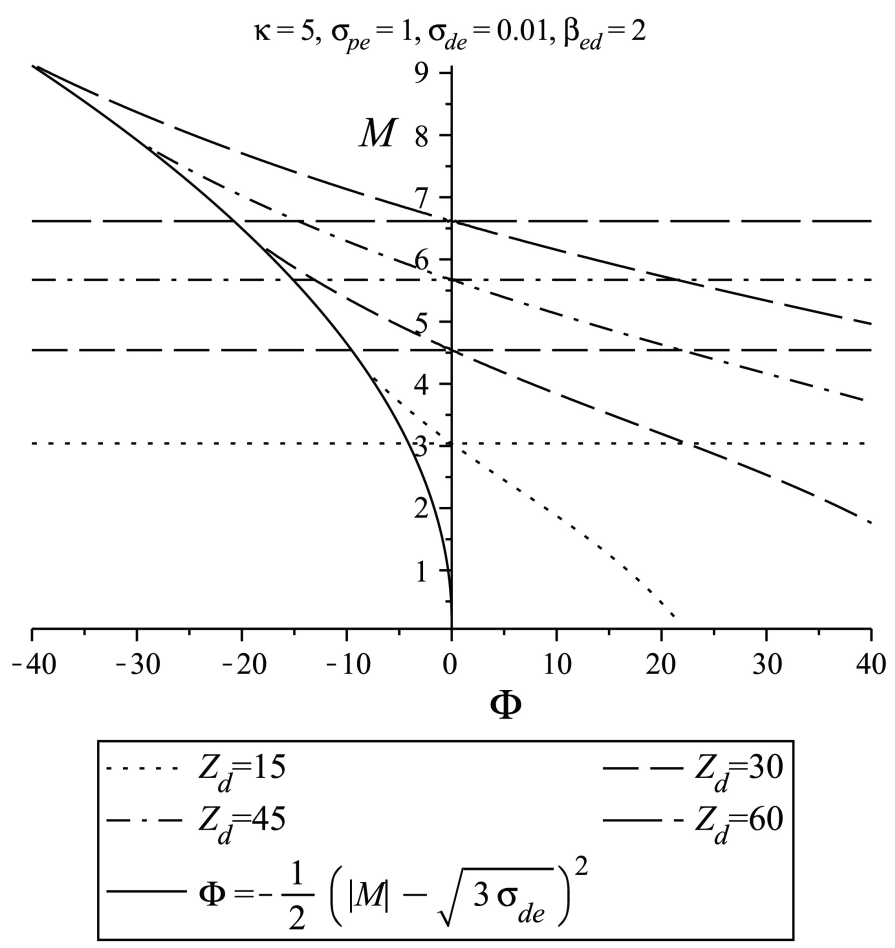

Fig. 2a

Fig. (2a). Same as in Fig. (1a), for $\beta_{e d}=2$ and $\kappa=5$. 


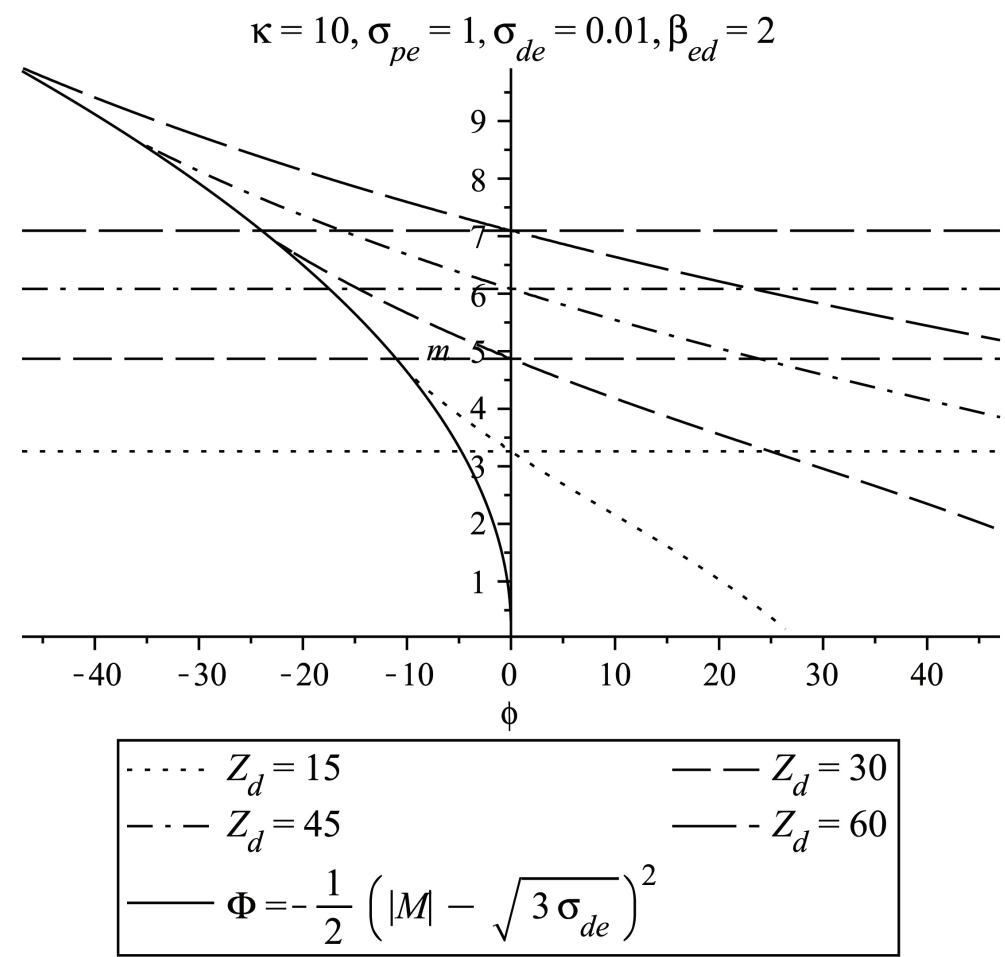

Fig. 2b

Fig. (2b). Same as in Fig. (1a), for $\beta_{e d}=2$ and $\kappa=10$.

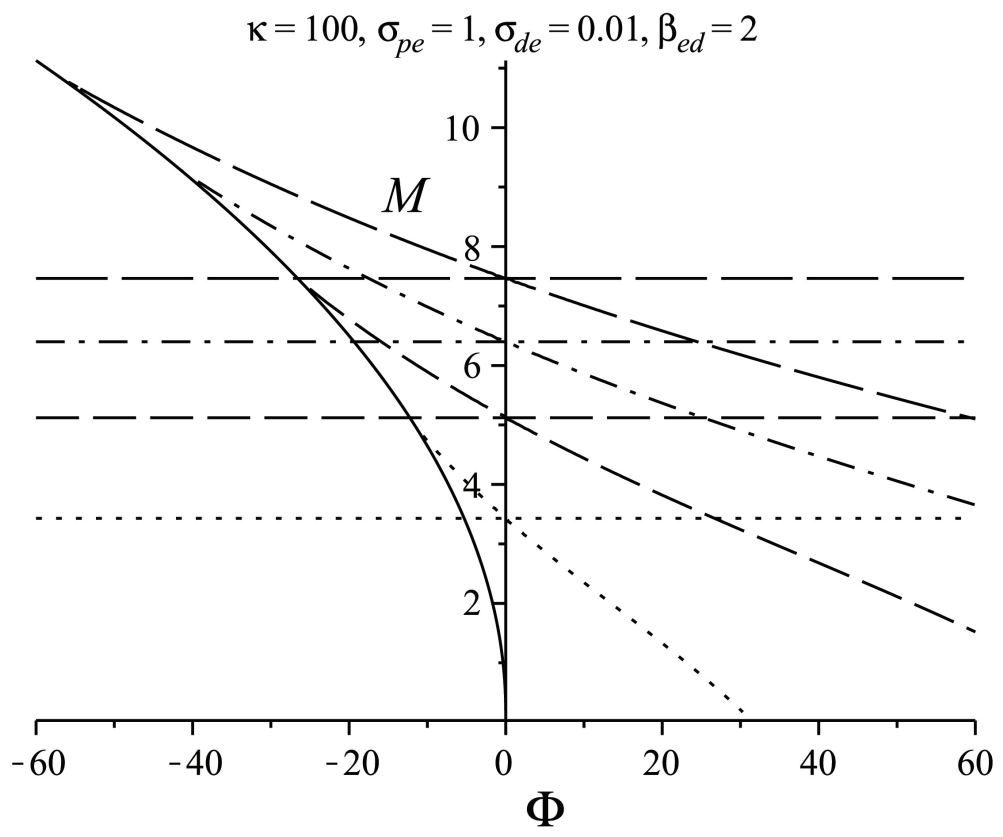

$$
\begin{array}{lr}
\cdots Z_{d}=15 & --Z_{d}=30 \\
-\cdots Z_{d}=45 & --Z_{d}=60 \\
-\Phi=-\frac{1}{2}\left(|M|-\sqrt{3 \sigma_{d e}}\right)^{2} &
\end{array}
$$

Fig. 2c

Fig. (2c). Same as in Fig. (1a), for $\beta_{e d}=2$ and $\kappa=100$. 


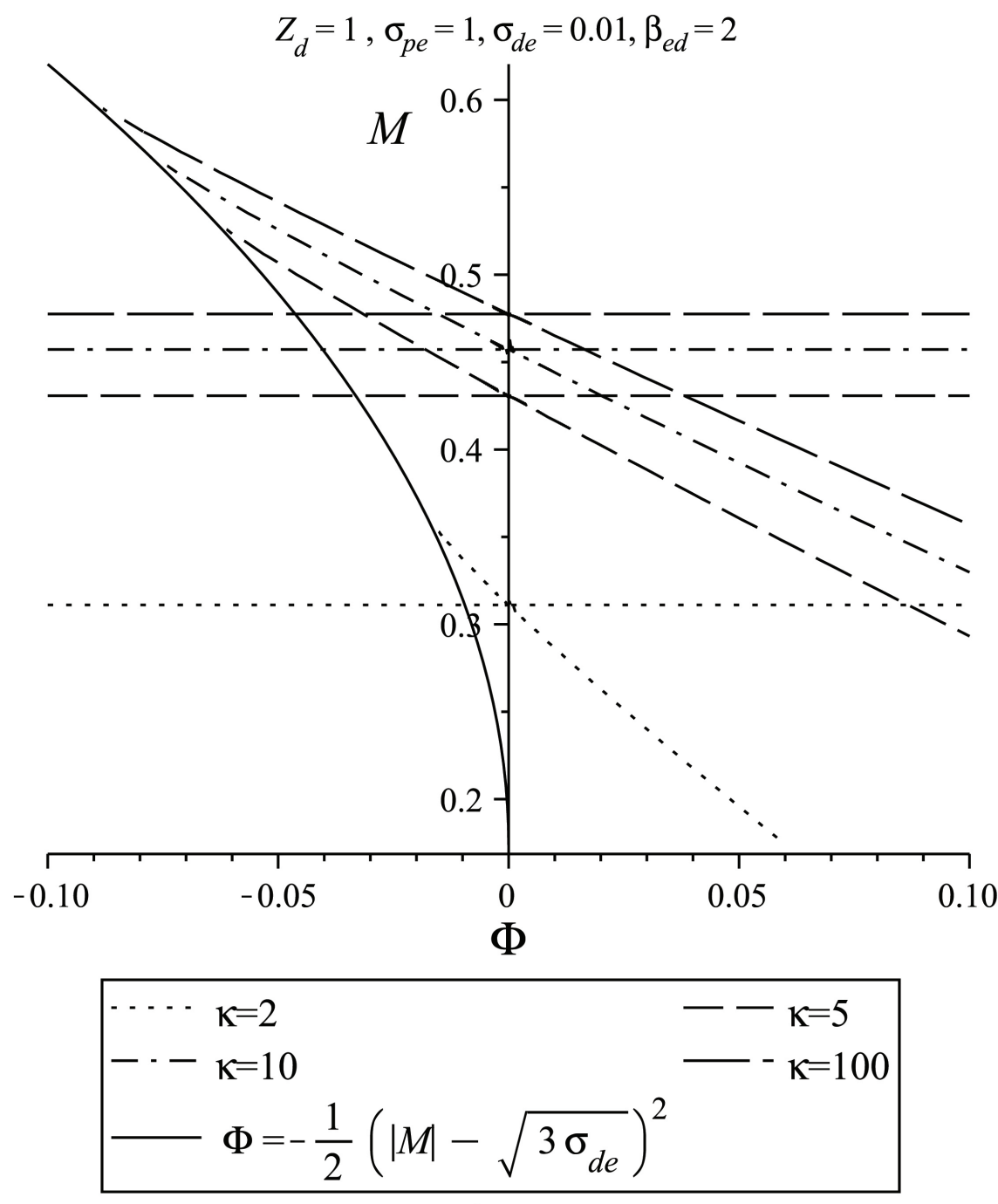

Fig. 3

Fig. (3). The contours of $\Phi=\frac{-1}{2}\left(|M|-\sqrt{3 \sigma_{d e}}\right)^{2}$ (solid curve), $\psi(\Phi)=0$ (doted, dashed, dot-dashed and long dashed curves for $\kappa=2, \kappa=5, \kappa=10$ and $\kappa=100$, respectively) and $\psi^{\prime \prime}(0)=0$ (doted, dashed, dot-dashed and long dashed horizontal lines for $\kappa=2, \kappa=5, \kappa=10$ and $\kappa=100$ respectively) in the $\Phi-M$ plane. The other plasma parameters set to $\sigma_{d e}=0.01, \sigma_{p e}=1, \beta_{e d}=2$ and $Z_{d}=1$.

dashed, dot-dashed and long dashed curves for various values of $Z_{d}$ and the contour $\psi^{\prime \prime}(\Phi=0)=0$ with dotted, dashed, dot-dashed and long dashed horizontal lines for the same values of $Z_{d}$. Knowing that the condition $\psi(\Phi, M)=0$ indicates the nontrivial root of the pseudopotential $\psi(\Phi, M)$ or the maximum value of amplitude, i.e., $\Phi_{m}$, we may find that the regions below the contour $\psi(\Phi, M)=0$ in the $(\Phi, M)$ plane indicate the formation of a typical potential well in which $\psi(\Phi, M)<0$. On the other hand, knowing that the condition $\psi^{\prime \prime}(\Phi=0)<0$ defines the threshold Mach number $M$, at which localized pulses can propagate in the plasma, we can find that the region above the contour $\psi^{\prime \prime}(\Phi=0)=0$ in the $(\Phi, M)$ plane indicate the frormation of a typical potential well. So, we may discuss the allowed regions for the existence of dust-acoustic solitons via these graphs.

It is remarked that both of the acceptable minimum and maximum Mach numbers increase as dust charge $Z_{d}$ increases. Also, it is observed that only negative potential pulses can propagate and their amplitudes increase as the Mach number $M$ increases (this is in contrast to the result given in Ref. [37] for an electron-positron-ion plasma).

(Fig. (1a)) shows the contours of $\Phi=-1 / 2\left(|M|-\sqrt{3 \sigma_{d e}}\right)^{2}$ (solid curve), $\psi(\Phi, M)=0$, (dotted, dashed, dot-dashed and long dashed curves for $Z_{d}=15, Z_{d}=30 \mathrm{Z}_{\mathrm{d}}=30, Z_{d}=45$ and $Z_{d}=60$, respectively) and $\psi^{\prime \prime}(\Phi=0)=0 \quad$ (dotted, 


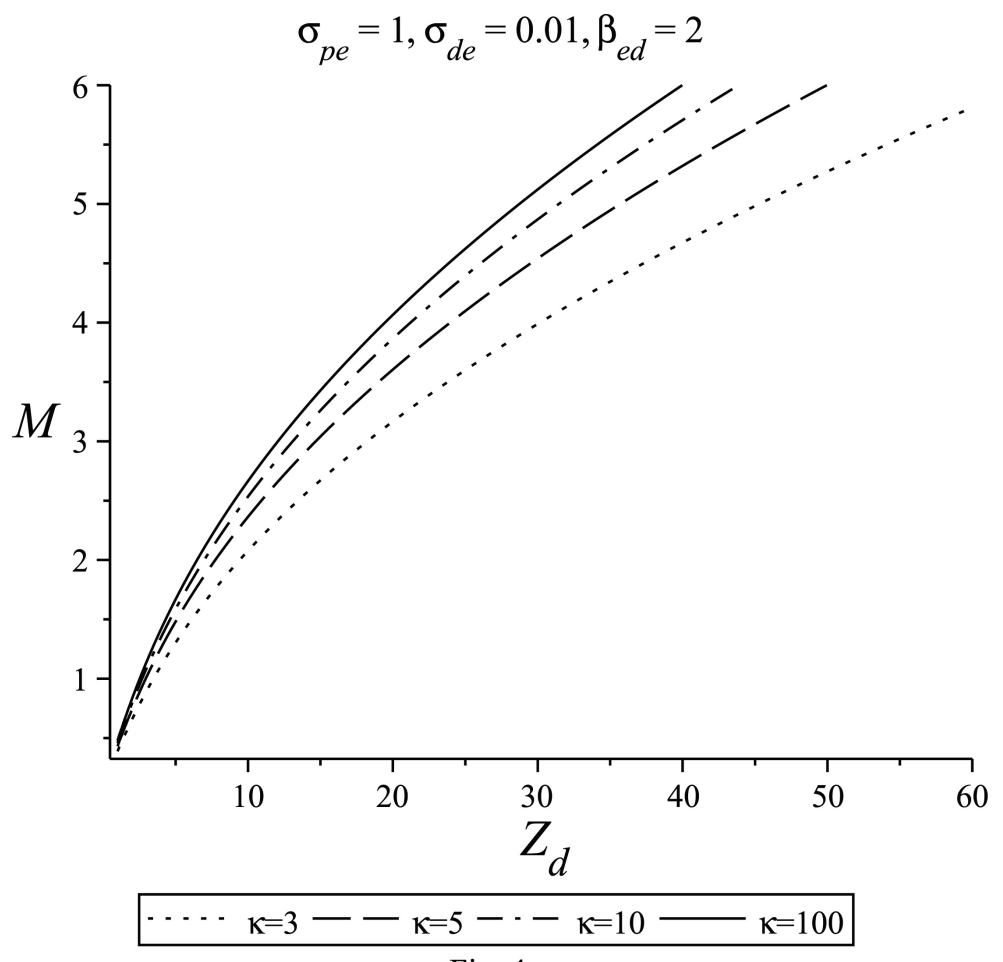

Fig. 4

Fig. (4). the contours of $\psi^{\prime \prime}(\Phi=0)=0$ in the $Z_{d}-M$ plane (doted, dashed, dot-dashed and solid curves for $\kappa=3, \kappa=5, \kappa=10$ and $\kappa=100$, respectively) with $\sigma_{p e}=1, \sigma_{d e}=0.01, \beta_{e d}=2$. Each curve indicates the threshold Mach number $M$ above which the solitons are allowed.

dashed, dot-dashed and long dashed horizontal lines for $Z_{d}=15, Z_{d}=30, Z_{d}=45$ and $Z_{d}=60$, respectively) in the plane $(\Phi, M)$. The other plasma parameters have been considered as $\sigma_{d e}=0.01, \sigma_{p e}=1, \beta_{e d}=2$ and $\kappa=2$. One must note that, $\psi(\Phi, M)$ is negative (positive) for values of $\Phi$ and $M$ which are to be chosen from upper (lower) relevant curves, whereas, it is complex for values $\Phi$ and $M$ under solid curve in Fig. (1a).

To inspect the effect of the fractional density of electron to dust, $\beta_{e d}$, on the existence regions of large amplitude solitons and their amplitude, we have depicted Figs. (1a-c) similar to Fig. (1a), for $\beta_{e d}=5$ and 10, respectively. Furthermore, it is found that the minimum/maximum Mach number increases as the dust charge $Z_{d}$ increases for the fixed $\beta_{e d}$, whereas, it decreases as $\beta_{e d}$ increases for the fixed $Z_{d}$.

In order to inspect the effect of the spectral index $K$ on the existence regions of solitary waves and on their amplitudes, we have depicted Figs. (2a-c) for $\kappa=5, \kappa=10$ and $\kappa=100$, respectively. The values of the other parameters and the description of various curves in Figs. (2a-c) are similar to Fig. (1a). We see that the minimum/maximum
Mach number increases and reaches to a finite value (in fact, to the Maxwellian limit) as the spectral index $\kappa$ increases. This is in agreement with the result given in Ref. [37] for the case of an electron-positron-ion plasma.

Until now, we remarked that only supersonic solitons with $M>1$ may propagate in the plasma for large values of $Z_{d}$. Now, let us consider a dusty plasma in which dust particles are fullerene ions with $Z_{d}=1$ [53]. In Fig. (3), we have plotted the contours of $\Phi=-1 / 2\left(|M|-\sqrt{3 \sigma_{d e}}\right)^{2}$ (solid curve), $\psi(\Phi, M)=0$ (dotted, dashed, dot-dashed and long dashed curves for $\kappa=2, \kappa=5, \kappa=10$ and $\kappa=100$, respectively) and $\psi^{\prime \prime}(\Phi=0)=0$ (dotted, dashed, dot-dashed and long dashed horizontal lines for $\kappa=2, \kappa=5, \kappa=10$ and $\kappa=100$, respectively) in the $(\Phi, M)$ plane. The other plasma parameters are $\sigma_{d e}=0.01, \sigma_{p e}=1, \beta_{e d}=2$. We find that in the case of fullerene ions with $Z_{d}=1$, only the subsonic negative solitons can propagate in the plasma, i.e., solitons with $M<1$. In fact, there is a critical value for $Z_{d}$ above/below which the supersonic/subsonic solitons can propagate in the plasma. In order to show the effect of dust charge $Z_{d}$ on the threshold Mach number $M$, in Fig. (4), we have numerically plotted the contours of $\psi^{\prime \prime}(\Phi=0)=0$ 


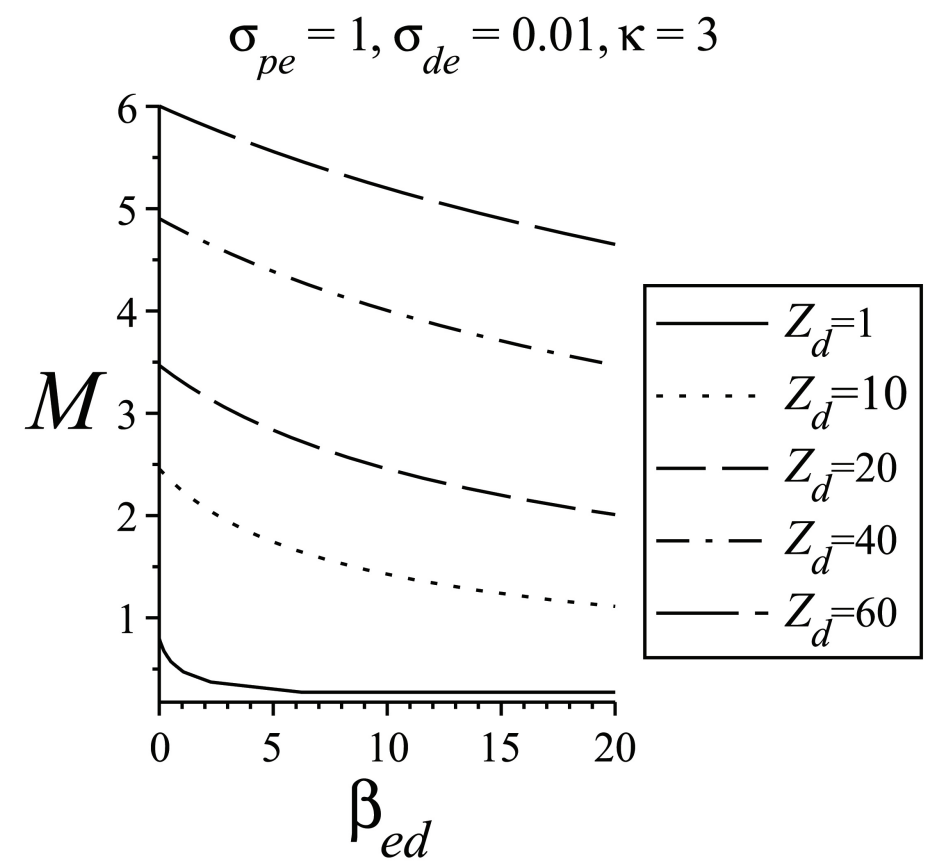

Fig. 5

Fig. (5). Variation of the threshold Mach number Mach Number $M$ with ratio of density of electron to dust ( $\left.\beta_{e d}\right)$ for different values of dust charge $\left(Z_{d}\right)$; the other plasma parameters are set to $\sigma_{p e}=1, \sigma_{d e}=0.01, \kappa=3$.

for some values of $\kappa$ in the $Z_{d}-M$ plane, with $\sigma_{p e}=1, \sigma_{d e}=0.01, \beta_{e d}=2$. It is pointed out that the condition $\psi^{\prime \prime}(\Phi=0)<0$ (threshold Mach number $M$, above which the solitons can propagate) is satisfied only above the curves in Fig. (4). It is found that an increase in the dust charge $Z_{d}$ increases the threshold Mach number $M$. In addition, Fig. (4) reveals that depend on the values of $Z_{d}$ both of the subsonic and supersonic solitons are allowed in the plasma. Furthermore, we have plotted the threshold Mach number $M$ versus the fractional density of electron to dust $\beta_{e d}$ in Fig. (5) for some values of $Z_{d}$ and with $\sigma_{p e}=1, \sigma_{d e}=0.01, \kappa=3$. We see that the threshold Mach number $M$ increases as the dust charge $Z_{d}$ increases and it decreases with $\beta_{e d}$.

It is to be mentioned that in ordinary plasmas, we usually consider a hydrogen plasma in which the ions are positively charged with $Z_{i}=1$. In such systems the positive ions constitute the background of the plasma, in which the ionacoustic solitons can propagate with a positive potential polarity, i.e., the compressive solitons. However, in this study we have considerd a quite different system comprising electron-positron pairs and negatively charged dust grains which are allowed to acquire a large negative charge with $Z_{d} \geq 1$. It is obvious that the features of the acoustic modes (here, the dust-acoustic solitons) should be modified in comparison with that of in ordinary plasmas. It is logical that in a negatively charged background, via dust particles, the dustacoustic solitons propagate with a negative potential polarity, i.e., rarefactive solitons. Here, we have no dust-acoustic structure with positive potential (compressive solions). On the other hand, in such plasma systems in which the negative charge of dusts lies in a wide range $\left(Z_{d}=1\right.$ to e.g., $Z_{d}=60$ ), a typical nonlinear dust-acoustic wave may possess small or sufficiently large amplitudes. It is reminded that in the nonlinear theory of plasma waves the soliton speed is proportional to the soliton amplitude. So, in such plasma systems the solitons are possible in a wide range of the Mach number from subsonic to supersonic.

Here, it is found that the Mach number carries large magnitude ( $M>1.6)$ for large values of the dust charge $Z_{d}$. The Mach numbers larger than 1.6 have been reported in a plasma consisting of inertial dust particles and inertial-less ions by Shchekinov [54] and also in dusty plasmas with inertial dust fluid and Boltzmann distributed electrons, positrons, and ions by Esfandyari-Kalejahi et al. [9]. However, the values of dust charge $Z_{d}$ may be very large in space plasmas [55] and consequently, the DASWs can be supersonic in these environments. 


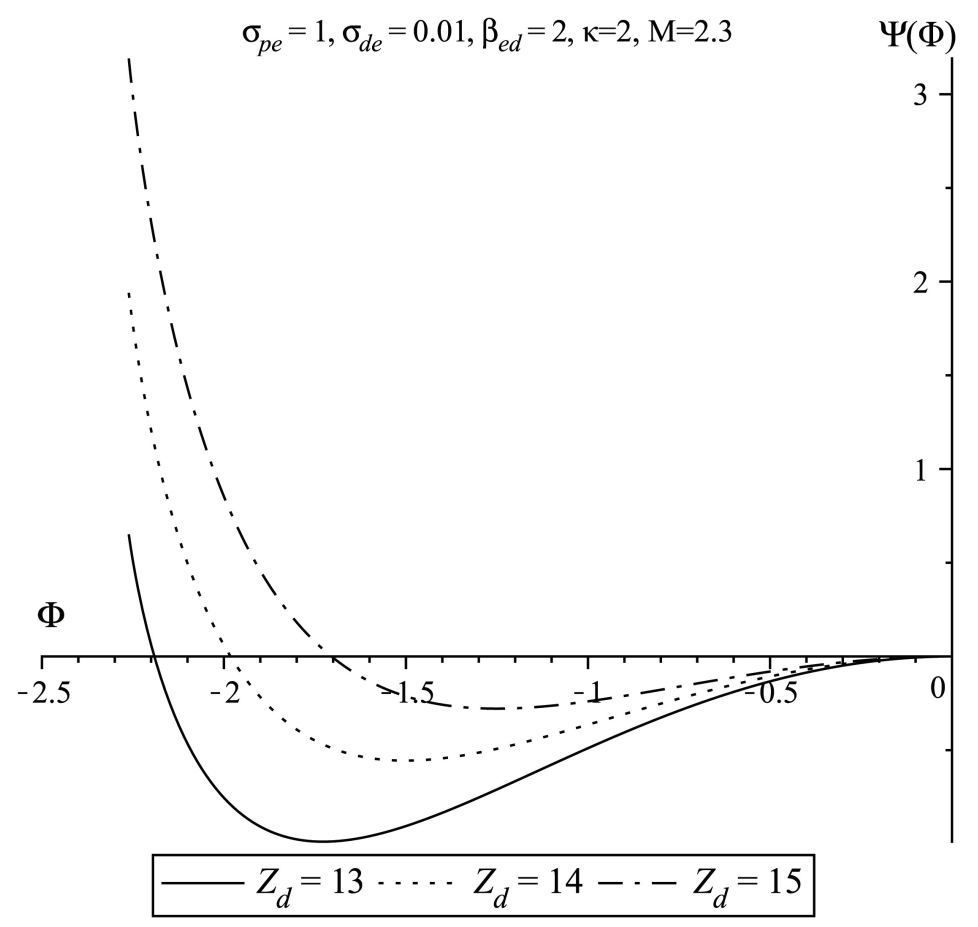

Fig. 6a

Fig. (6a). Variation of pseudo-potential $\psi(\Phi)$ versus potential $\Phi$ for different values of dust charge $\left(Z_{d}\right)$. The other plasma parameters are set to $\sigma_{p e}=1, \sigma_{d e}=0.01, \beta_{e d}=2, \kappa=2$ and $M=2.3$.

Now, we shall numerically investigate the effect of various parameters on the structure of potential well. It is reminded that in the pseudo-potential theory the shape of soliton pulse can be predicted via the analysis of pseudopotential function. The root of the pseudo-potential function $\psi(\phi)$ (width of potential well) corresponds to the maximum pulse amplitude $\left(\Phi_{m}\right)$, while the depth of the potential well is associated with the slope of soliton pulse $\Phi(\xi)$ (see Eq. (16)). So a deeper and wider potential well implies a steeper (narrower) and higher soliton pulse, and vice versa. In Fig. (6a), we have depicted numerically the variation of the pseudo-potential $\psi(\phi)$ with respect to the dust charge $Z_{d}$. It is found that with increasing the dust charge $Z_{d}$ the width and depth of potential well decrease, resulting in wider solitons (less steepness) with smaller amplitude. Fig. (1b) show the variation of the pseudo-potential $\psi(\phi)$ versus Mach number $M$. This figure indicates that increasing the soliton speed cause the width and depth of potential well to increase. So, the amplitude and the steepness of soliton profile increase with an increase in soliton speed. To show the effect of superthermality, in Fig. (6c) we have plotted variation of the pseudo-potential $\psi(\Phi)$ with respect to the superthermality index $\kappa$. It is found that the width and depth of potential well increase monotonically as a result of an increase in superthermality (i.e., a decrease in the value of $\kappa$ ). So, the maximum amplitude of the DA solitons $\left(\Phi_{m}\right)$ increases and the soliton pulse becomes steeper for a greater excess of superthermal particles.

The physical role played by the superthermal electrons and positrons may be explained as follows. Physically, in a superthermal plasma modeled by the Kappa-like distribution, the particles have distributed in a wider spectrum of the velocities, in comparison with a Maxwellian plasma. In such a model the spectral index $\kappa$ determines the deviations from the the Maxwellian distribution. In fact, the low values of the spectral index $\kappa$ correspond to a large fraction of superthermal particle populations in the plasma. Numerically plotting the velocity distribution function $f_{\kappa}(v)$ shows that in the case of a Kappa distribution (small values of $\kappa$ ), comparing with the Maxwellian one (sufficiently high values of $\kappa$, e.g., $\kappa=100$ ), there are more superthermal particles, i.e., the particles with the velocity faster than the thermal speed $v_{t h}=\left(2 K_{B} T / m\right)^{1 / 2}$ [56]. So, we expect that in the case of a plasma in which the distribution modelled by small values of $\kappa$, the dust-acoustic solitons have to be affected increasingly by the superthermal electrons and positrons. Here, the diagrams in Fig. (6c) confirm that for smaller values of $\kappa$ (more superthermality) the maximum amplitude has larger values relative to higher values of $\kappa$ (less superthermality).

Another result is that double layers cannot exist in the desired plasma system. This is because $d \psi /\left.d \Phi\right|_{\Phi=\Phi_{m}}$ cannot 


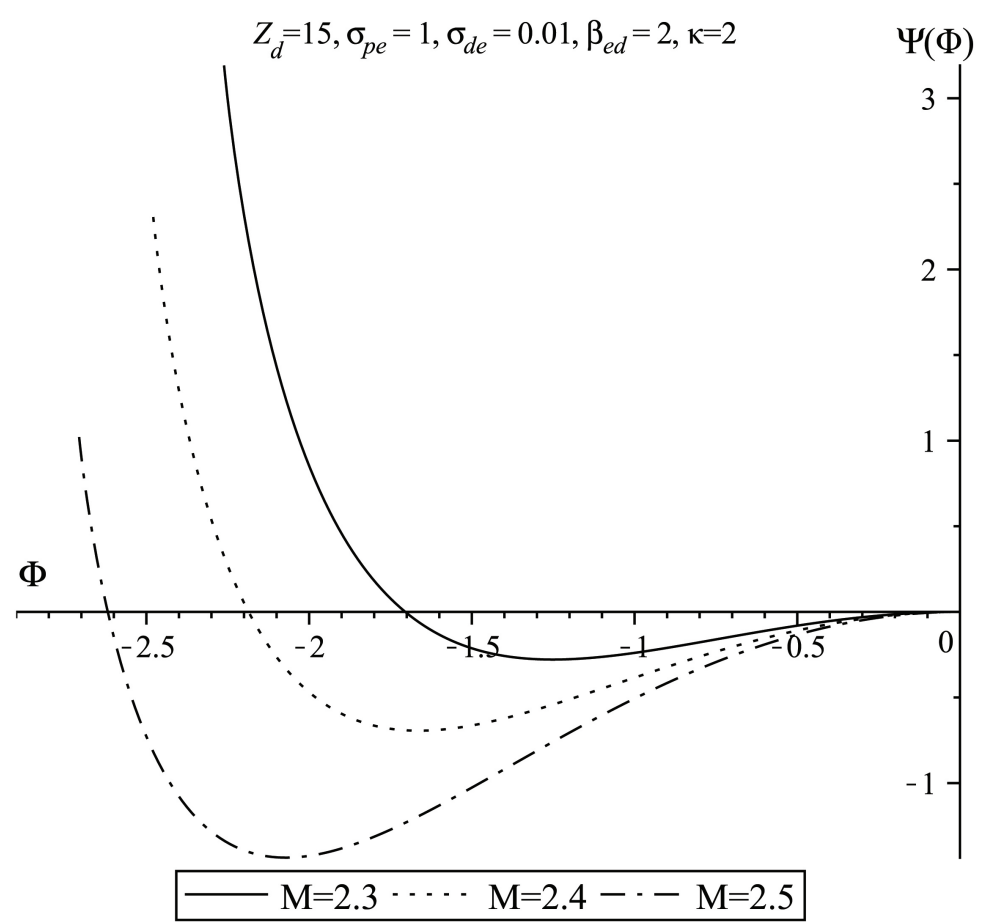

Fig. $6 \mathrm{~b}$

Fig. (6b). Variation of pseudo-potential $\psi(\Phi)$ versus potential $\Phi$ for different values of Mach number ( $M$ ). The other plasma parameters are set to $\sigma_{p e}=1, \sigma_{d e}=0.01, \beta_{e d}=2, \kappa=2$ and $Z_{d}=15$.

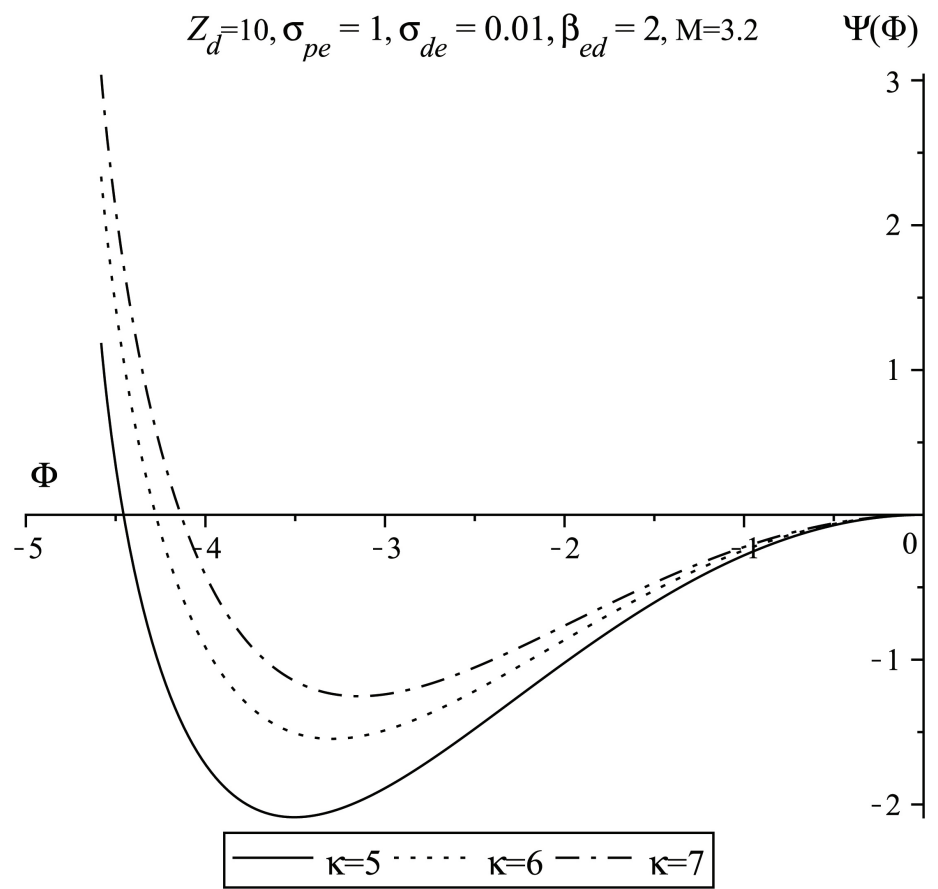

Fig. 6c

Fig. (6c). Variation of pseudo-potential $\psi(\Phi)$ versus potential $\Phi$ for different values of spectral index $(\mathcal{K})$. The other plasma parameters are set to $Z_{d}=10, \sigma_{p e}=1, \sigma_{d e}=0.01, \beta_{e d}=2$ and $M=3.2$.

be zero (the condition $d \psi /\left.d \Phi\right|_{\Phi=\Phi_{m}}=0$ is necessary in order to formation the double layers). Physically, we may discuss the reasons that double layers do not exist. "A double layer is a structure in a plasma which consists of two parallel layers with opposite electrical charge. Generally, there are two different kinds of double layers, which are formed differently; current carrying double layers and current-free double layers. Current carrying double layers may arise in plasmas carrying a current. In this case, the necessary condition for the for- 
mation of double layers is that the electron drift velocity exceeds the electron thermal velocity, i.e., double layers are a result of the Buneman instability. On the other hand, currentfree double layers occur at the boundary between plasma regions with different plasma properties, where quasineutrality is violated" [57-60]. In order to clarify the situation in which the current-free double layers may occur please note the following discussion. "Consider a plasma divided into two regions by a plane, which has a higher electron temperature on one side than on the other (the same analysis can also be done for different densities). This means that the electrons on one side of the interface have a greater thermal velocity. The electrons may stream freely in either direction, and the flux of electrons from the hot plasma to the cold plasma will be greater than the flux of the electrons from the cold plasma to the hot plasma, because the electrons from the hot side have a greater average speed. Because many more electrons enter the cold plasma than exit it, part of the cold region becomes negatively charged. The hot plasma, conversely, becomes positively charged. Therefore, an electric field builds up, which starts to accelerate electrons towards the hot region, reducing the net flux. In the end, the electric field builds up until the fluxes of electrons in either direction are equal, and further charge build up in the two plasmas is prevented. The potential drop is in fact exactly equal to the difference in thermal energy between the two plasma regions in this typical case" [57-60]. Now, it is found that in this study in which there is no drift velocity and no current in the plasma, and also there are not two electron (positron) species with different temperatures, a potential drop could not be happened and double layers do not exist.

\section{CONCLUSION}

In this paper we have investigated the propagation of large amplitude DA solitary waves in a three-component plasma consisting of electrons, positrons and dust particulates using the Sagdeev pseudo-potential method. In order to provide a better fit with the experimental observations, in particular for space plasmas, we have assumed that the electrons and positrons are superthermal which modeled by a $\kappa$ -like distribution rather than Maxwellian one.

It is remarked that both the acceptable minimum and maximum Mach numbers increase as the dust charge $Z_{d}$ increases. Also, it is found that only negative potential structures can propagate and their amplitudes increase as Mach number $M$ increases. Moreover, we have numerically shown that there is a critical value for dust charge $Z_{d}$ below/above which the subsonic/supersonic solitary waves can exist. Furthermore, it has been seen that in the case in which the dust particles are negative fullerene ions, $Z_{d}=1$, the subsonic dust acoustic solitons can also propagate in the plasma. Another result is that the double layers cannot occur in the plasma.

\section{CONFLICT OF INTEREST}

The authors confirm that this article content has no conflict of interest.

\section{ACKNOWLEDGEMENTS}

Declared none.

\section{REFERENCES}

[1] Goertz CK. Dusty plasmas in the solar system. Rev Geophys 1989; 27: 271-92.

[2] Mendis DA. Progress in the study of dusty plasmas. Plasma Sources Sci Technol 2002; 11: A219.

[3] Mendis DA, Rosenberg M. Cosmic dusty plasma. Annu Rev Astron Astrophys 1994; 32: 419-63.

[4] Horanyi M. Charged dust dynamics in the solar system Annu Rev Astron Astrophys 1996; 34: 383-418.

[5] Verheest F. Waves in dusty space plasmas. Dordrecht: Kluwer Academic Publishers 2000.

[6] Tajima T, Shibata K. Plasma astrophysics. New York: AddisonWesley 1997.

[7] Ditmire T, Tisch JWG, Springate E, et al. High-energy ions produced in explosions of superheated atomic clusters. Nature 1997; 386: 54-6.

[8] Ditmire T, Tisch JWG, Springate E, et al. High energy ion explosion of atomic clusters: Transition from molecular to plasma behavior. Phys Rev Lett 1997; 78: 2732 -5.

[9] Liu CS, Tripathi VK. Ion coulomb explosion of clusters by a Gaussian laser beam. Phys Plasmas 2003; 10: 4085.

[10] Mourou GA, Barty CPJ, Perry MD. Ultrahigh-intensity lasers: Physics of the extreme on a tabletop. Phys Today 1998; 51: 22-8.

[11] Washimi H, Taniuti T. Propagation of ion-acoustic solitary waves of small amplitude. Phys Rev Lett 1966; 17, 996.

[12] Sagdeev RZ. Cooperative phenomena and shock waves in collisionless plasmas. In: Leontovich MA, Ed. Rev Plasma Phys. New York: Consultants Bureau 1966; 44: p. 23.

[13] Ivlev AV, Morfill G. Dust acoustic solitons with variable particle charge: Role of the ion distribution. Phys Rev E 2001; 63: 026412.

[14] Popel SI, Kopnin SI, Kosarev IN, Yu MY. Solitons in earth's dusty mesosphere. Adv Space Res 2006; 37(2): 414-9.

[15] Shukla PK, Farid T, Stenflo L, Onishchenko OG. Sheared-flowdriven vortices in a magnetized dusty electron-positron plasma. J Plasma Phys 2000; 64: 427-31.

[16] Mirza AM, Khan K. Self-organized and chaotic states in dustcontaminated electron-positron magnetoplasma with equilibrium flows. Phys Scr 2002; 66: 376.

[17] Esfandyari-Kalejahi A, Kourakis I, Mehdipoor M, Shukla PK Electrostatic mode envelope excitations in $\mathrm{e}-\mathrm{p}-\mathrm{i}$ plasmasapplication in warm pair ion plasmas with a small fraction of stationary ions. J Phys A Math Gen 2006; 39: 13817.

[18] Rao NN, Shukla PK, Yu MY. Dust-acoustic waves in dusty plasmas. Planet Space Sci 1990; 38: 543-6.

[19] Verheest F. Nonlinear dust-acoustic waves in multispecies dusty plasmas. Planet Space Sci 1992; 40(1): 1-6.

[20] Barkan A, Merlino RL, D'Angelo N. Laboratory observation of the dust-acoustic wave mode. Phys Plasmas 1995; 2: 3563.

[21] Esfandyari-Kalejahi A, Afsari-Ghazi M, Noori K, Irani S. Large amplitude dust-acoustic solitary waves in electron-positron-ion plasma with dust grains. Phys Plasmas 2012; 19: 082308.

[22] Mace RL, Baboolal S, Bharuthram R, Hellberg MA. Arbitraryamplitude electron-acoustic solitons in a two-electron-component plasma. J Plasma Phys 1991; 45: 323-38.

[23] Singh SV, Lakhina GS. Generation of electron-acoustic waves in the magnetosphere. Planet Space Sci 2001; 49: 107-14.

[24] Lakhina GS, Kakad AP, Singh SV, Verheest F. Ion- and electronacoustic solitons in two-electron temperature space plasmas. Phys Plasmas 2008; 15: 062903. 
[25] Singh SV, Lakhina GS. Electron acoustic solitary waves with nonthermal distribution of electrons. Nonl Proc Geophys 2004; 11: 275-9.

[26] Steinacker J, Miller JA. Stochastic gyroresonant electron acceleration in a low-beta plasma: Interaction with parallel transverse cold plasma waves. Astrophys J 1992; 393: 764-81.

[27] (a) Krapchev VB, Ram AK. Adiabatic theory for a single nonlinear wave in a vlasov plasma. Phys Rev A 1980; 22: 1229; (b) Kiran Z, Shah HA, Qureshi MNS, Murtaza G. Parallel proton heating in solar wind using generalized $(r, q)$ distribution Function. Solar Phys 2006; 236:167-83.

[28] (a) Luque A, Schamel H. Electrostatic trapping as a key to the dynamics of plasmas, fluids and other collective systems. Phys Rep 2005; 415: 261-359; (b) Lifshitz EM, Pitaevskii LP. Physical Kinetics. New York: Pergamon 1975.

[29] Vasyliunas VM. A survey of low-energy electrons in the evening sector of the magnetosphere with OGO 1 and OGO 3. J Geophys Res 1968; 73: 2839-84.

[30] Sultana S, Kourakis I, Saini NS, Hellberg MA. Oblique electrostatic excitations in a magnetized plasma in the presence of excess superthermal electrons. Phys Plasmas 2010; 17: 032310.

[31] Saini NS, Kourakis I. Electron beam-plasma interaction and ionacoustic solitary waves in plasmas with a superthermal electron component. Plasma Phys Controlled Fusion 2010; 52: 075009.

[32] Saini NS, Kourakis I, Hellberg MA. Arbitrary amplitude ionacoustic solitary excitations in the presence of excess superthermal electrons. Phys Plasmas 2009; 16: 062903.

[33] Devanandhan S, Singh SV, Lakhina GS, Bharuthram R. Electron acoustic solitons in the presence of an electron beam and superthermal electrons. Non Proc Geophys 2011; 18: 627-34.

[34] Gogoi R, Roychoudhury R, Khan M. Arbitrary amplitude dust ion acoustic solitary waves and double layers in a kappa distributed electron plasma. Ind J Pure Appl Phys 2011; 49: 173-9.

[35] El-Bedwehy NA, Moslem WM. Zakharov-Kuznetsov-Burgers equation in superthermal electron-positron-ion plasma. Astrophys Space Sci 2011; 335(2): 435-42.

[36] Hussain S, Akhtar N, Mahmood S. Ion acoustic solitons in negative ion plasmas with superthermal electrons. Astrophys Space Sci 2012; 338: 265-70.

[37] El-Awady EI, El- Tantawy SA, Moslem WM, Shukla PK. Electron-positron-ion plasma with kappa distribution: Ion acoustic soliton propagation. Phys Lett A 2010; 374: 3216-9.

[38] Sabry R, Moslem WM, Shukla PK. On the generation of envelope solitons in the presence of excess superthermal electrons and positrons. Astrophys Space Sci 2011; 333: 203-8.

[39] Meyer-Vernet N. How does the solar wind blow? Some basic aspects. In: Bothemer V, Hady AA, Eds. Solar activity and its magnetic origin. Proceeding IAU Symposium No. 233; 2006 March 31Apr 3; Cairo, Egypt; pp. 269-76.

[40] Summers D, Thorne RM. The modified plasma dispersion function. Phys Fluids B 1991; 3(8): 1835.

[41] Mace RL, Hellberg MA. A dispersion function for plasmas containing superthermal particles. Phys Plasmas 1995; 2: 2098.
[42] Hellberg MA, Mace RL, Cattaert T. Effects of superthermal particles on waves in magnetized space plasmas. Space Sci Rev 2005; 121: $127-39$.

[43] Feldman WC, Anderson RC, Asbridge JR, Bame SJ, Gosling JT, Zwickl RD. Plasma electron signature of magnetic connection to the Earth's bow shock: ISEE 3. J Geophys Res 1982; 87: 632-42.

[44] Pierrard V, Lemair JF. Lorentzian ion exosphere model. J Geophys Res 1996; 101: 7923-34.

[45] Hellberg MA, Mace RL, Armstrong RJ, Karlstad G. Electronacoustic waves in the laboratory: An experiment revisited. J Plasma Phys 2000; 64: 433-43.

[46] Baluku TK, Hellberg MA. Dust acoustic solitons in plasmas with kappa-distributed electrons and/or ions. Phys Plasmas 2008; 15: 123705.

[47] Chen FF. Introduction to plasma physics and controlled fusion. New York: Plenum Press 1984.

[48] Daun WS. The Kadomtsev-Petviashvili (KP) equation of dust acoustic waves for hot dust plasmas. Chaos Soliton Fract 2002; 14: 503-6.

[49] Moslem WM, Sabry R, El-Labany SK, Shukla PK. Dust-acoustic rogue waves in a nonextensive plasma. Phys Rev E 2011; 84: 066402.

[50] Gill TS, Bedi C, Bains AS. Ion acoustic shock waves in weakly relativistic multicomponent quantum plasma. Phys Scr 2010; 81: 055503.

[51] Berthomier M, Pottelette R, Malinger M, Khotyaintsev Y. Electron-acoustic solitons in an electron-beam plasma system. Phys Plasmas 2000; 7: 2987.

[52] Treumann RA, Baumjohann W. Advanced space plasma physics. London: Imperial Colleage Press 1997.

[53] (a) Oohara W, Hatakeyama R. Pair-ion plasma generation using fullerenes. Phys Rev Lett 2003; 91: 205005; (b) Oohara W, Date D, Hatakeyama R. Electrostatic waves in a paired fullerene-ion plasma. Phys Rev Lett 2005; 95: 175003; (c) Hatakeyama R, Oohara W. Properties of pair-ion plasmas using fullerenes. Phys Scr 2005; 116: 101 .

[54] Shchekinov YA. Dust-acoustic soliton with nonisothermal ions. Phys Lett A 1997; 225:117-20.

[55] Kotsarenko NY, Koshevaya SV, Kotsarenko AN. Dusty plasma in space. Geofísica Internacional 1998; 37: 71-86.

[56] Saberian E, Esfandyari-Kalejahi A, Afsari-Ghazi M, RastkarEbrahimzadeh A. Propagation of ion-acoustic solitons in an electron beam-superthermal plasma system with finite ion-temperature: Linear and fully nonlinear investigation. Phys Plasmas 2013; 20: 032307.

[57] Block LP. Potential double layers in the ionosphere. Cosmic Electrodyn 1972; 3(1): 349-57.

[58] Knorr G, Goertz CK. Existence and stability of strong potential double layers. Astrophys Space Sci 1974; 31(2): 209-23.

[59] Goertz CK, Joyce G. Numerical simulation of the plasma double layer. Astrophys Space Sci 1975; 32(1): 165-73.

[60] DeGroot JS, Barnes C, Walstead AE, Buneman O. Localized structures and anomalous de resistivity. Phys Rev Lett 1977; 38(1): 1283.

Received: August 03, 2014

Revised: October 25, 2014

Accepted: December 09, 2014

(C) Saberian et al.; Licensee Bentham Open.

This is an open access article licensed under the terms of the Creative Commons Attribution Non-Commercial License (http://creativecommons.org/licenses/by-nc/3.0/) which permits unrestricted, non-commercial use, distribution and reproduction in any medium, provided the work is properly cited. 